\section{The cost of teaching an intern in New South Wales}

To the Editor: While Oates and colleagues ${ }^{1}$ provide an insightful introduction to intern teaching in New South Wales, I offer an alternative to their views; what is considered teaching is not agreed on, and does not necessarily translate to learning.

Informal teaching (and learning) occurs during work-based activities like ward rounds, departmental meetings, grand rounds and quality improvement activities. These are set out as learning opportunities for interns by the Australian Medical Council. $^{2}$

What supervisors consider to be informal learning probably does not coincide with interns' expectations of teaching.

Junior medical officers should realise that, for them, learning from informal teaching is not about acquiring knowledge of diseases or skills, as it is for medical students. Rather, it is about acquiring knowledge of work processes and resource management - expertise not well described in medical literature. Fiona Lake, a developer of Teaching on the Run,

bases her own teaching on the idea that if something can be learnt from a textbook, it is of no help for her to teach it as well. "It's a complete waste of time for me to teach it!"3

Adult learning forms a significant portion of any postgraduate vocational training and can occur by both a cognitive approach, based on andragogy theory, and an apprenticeship model, in which "learning by doing" and "master as role model" are the basis. ${ }^{4}$

Perhaps the main issue is not the perception of how teaching should be done, but how learning should occur.

Shyan LL Goh Orthopaedic Surgeon

Rockhampton Hospital, Rockhampton, QLD.

sgoh@hotmail.com

Competing interests: No relevant disclosures.

\section{doi: 10.5694/mjal4.00550}

1 Oates RK, Goulston KJ, Bingham CM, Dent OF. The cost of teaching an intern in New South Wales. Med J Aust 2014; 200: 100-103.

2 Australian Medical Council, Medical Board of Australia. Guide to intern training in Australia. 2013. http://www.amc.org.au/index.php/ar/ psa (accessed Jul 2014).

3 McGilvray A. Teaching in practice. Med J Aust 2013; 199 (7): Cl-C2

4 Swanwick T. Informal learning in postgraduate medical education: from cognitivism to 'culturism'. Med Educ 2005; 39: 859-865.

IN REPLY: We fully agree with Goh that teaching does not translate to learning. In our article, ${ }^{1}$ we were careful to concentrate on teaching, both formal and informal, received by interns, and not learning something that is much more difficult to measure.

We defined informal teaching as being spontaneous, non-timetabled and sporadic, pointing out that it may occur during a ward round, walking along a corridor or at the end of a consultation.

Like Goh, we acknowledged that teaching also occurs as a result of observation and practical experience, which were not included in our study. We emphasised that there is much more involved in intern education than the formal and informal teaching considered here, and we mentioned: observation by interns; self-learning; practical experience; self-reflection; and the influence of role models.

It may well be that some teaching occurred that was not identified as such by the interns surveyed. What is important is that formal and informal teaching should be acknowledged as a crucial part of the intern experience and that supervisors ensure that teaching and learning, in all its forms, is emphasised.

R Kim Oates Emeritus Professor

Kerry J Goulston Emeritus Professor

Sydney Medical School, University of Sydney, Sydney, NSW.

kim.oates@sydney.edu.au

Competing interests: No relevant disclosures.

doi: 10.5694/mjal4.00699

1 Oates RK, Goulston KJ, Bingham CM, Dent OF. The cost of teaching an intern in New South Wales. Med J Aust 2014; 200: 100-103.

what is considered teaching is not agreed on, and does not necessarily translate to learning

Goh

learning ... is much more difficult to measure

Oates et al

\section{Preventing peripheral intravenous catheter- associated bloodstream infection: the randomised controlled trial versus the real world}

To THE EdIToR: Debate regarding the prevention of peripheral intravenous catheter (PIVC)associated bloodstream infection has been enriched by research and letters published in the Journal. ${ }^{1-3}$ Stuart and colleagues and Collignon and colleagues highlighted an association of long dwell times with PIVCassociated bloodstream infection. ${ }^{1,2}$ Rickard and colleagues refer to contrasting high-level evidence in a Cochrane review. ${ }^{3,4}$ We would suggest that important lessons can be learnt from both perspectives.

The Hawthorne effect and strict inclusion and exclusion criteria mean that, while largely free of bias, the outcomes of clinical trials are not generalisable. The retrospective data on PIVCassociated bloodstream infections reported by Stuart et al and Collignon et al, while methodologically limited, demonstrate the real-world experience of hundreds of patients. ${ }^{1,2}$

We believe that clinicians should consider all forms of evidence when designing processes to ensure safe stewardship of PIVCs. In health services with independent "line teams" and a comprehensive PIVC insertion bundle, a"replace as clinically indicated" approach may be appropriate. Other health care services may be better suited to a mandatory replacement policy.

Given evidence that half of PIVCs inserted in our tertiary emergency department (ED) were unused, ${ }^{5}$ along with the evidence of PIVC-associated infection, ${ }^{1}$ Monash Health introduced a comprehensive multimodal change process to reduce unused PIVCs and 
subsequent bloodstream infections. Clinicians in Monash Health EDs are asked to only insert a PIVC if they feel it is at least $80 \%$ likely it will be used in the following 4 hours. An accompanying education campaign provides guidance in cases where a precautionary PIVC may be required, such as for patients who present after a seizure. The aim of the intervention is to avoid insertion of unnecessary PIVCs, while ensuring appropriate insertion, and to improve insertion methods. Four months after the intervention began, we demonstrated a reduction in total monthly PIVCs from 1413 to 928 and an unused PIVC rate of $19.3 \%$ (unpublished data). This change process has been offered to EDs throughoutVictoria by the Department of Health. ${ }^{6}$

We would support the establishment of a specific national standard regarding the stewardship of PIVCs, which should include the need to carefully evaluate whether insertion of the PIVC is necessary.

Diana Egerton-Warburton Director of Emergency Medicine Research,' and Adjunct Senior Lecturer ${ }^{2}$

George Braitberg Professor of Emergency Medicine Anthony Kambourakis Director of Emergency Medicine $^{1}$

1 Monash Health, Melbourne, VIC.

2 Southern Clinical School, Monash University, Melbourne, VIC.

diana.egertonwarburton@monashhealth.org

Competing interests: No relevant disclosures. doi: 10.5694/mjal4.00363

1 Stuart RL, Cameron DRM, Scott C, et al. Peripheral intravenous catheter-associated Staphylococcus aureus bacteraemia: more than 5 years of prospective data from two tertiary health services. Med J Aust 2013; 198: 551-553.

2 Collignon PJ, Kimber FJ, Beckingham WD, Roberts JL. Prevention of peripheral intravenous catheter-related bloodstream infections: the need for routine replacement [letter]. Med J Aust 2013; 199: 750-751.

3 Rickard CM, Webster J, Playford EG. Prevention of peripheral intravenous catheter-related bloodstream infections: the need for routine replacement [letter]. Med J Aust 2013; 199: 751-752.

4 Webster J, Osborne S, Rickard C, Hall J. Clinically-indicated replacement versus routine replacement of peripheral venous catheters. Cochrane Database Syst Rev 2010; (3): CD007798.

5 Limm El, Fang X, Dendle C, et al. Half of all peripheral intravenous lines in an Australian tertiary emergency department are unused: pain with no gain? Ann Emerg Med 2013; 62: 521-525. doi: 10.1016/j.annemergmed.2013.02.022.

6 Emergency Care Improvement and Innovation Clinical Network, Department of Health, Victoria Evidence-based care in emergency departments 2014. http://health.vic.gov.au/clinicalnetworks/ emergency/em2014-activities.htm (accessed Mar 2014).

\section{Ipilimumab-induced hypophysitis: early Australian experience}

To THE EdiTor: We report two men aged in their 60 s receiving ipilimumab for metastatic melanoma who presented with headache and constitutional symptoms after the third 3-weekly dose, and were diagnosed with ipilimumab-induced hypophysitis. Ipilimumab is a monoclonal antibody that binds to cytotoxic T lymphocyte-associated antigen 4, resulting in T-cell activation and proliferation. It was the first therapy to yield a survival benefit in metastatic melanoma, ${ }^{1}$ but at the cost of frequent immune-related adverse events. $^{2}$

Patient 1 experienced headache, fatigue, postural lightheadedness, anorexia and asthenia. Morning blood test results before steroid administration were consistent with central hypocortisolism (serum cortisol, $<28 \mathrm{nmol} / \mathrm{L}$ [reference interval (RI), 70$650 \mathrm{nmol} / \mathrm{L}]$; inappropriately normal adrenocorticotropic hormone [ACTH], $1.2 \mathrm{pmol} / \mathrm{L}$ [RI, 0-12.0 pmol/L]), hypothyroidism (low thyroid-stimulating hormone [TSH], 0.2 mIU/L [RI, 0.4-4.0 mIU/L]; low free thyroxine $\left[\mathrm{FT}_{4}\right], 8.9 \mathrm{pmol} / \mathrm{L}$ [RI, 9.0-19.0 pmol/L]; normal free triiodothyronine $\left[\mathrm{FT}_{3}\right], 4.7 \mathrm{pmol} / \mathrm{L}$ [RI, 2.6-6.0 pmol/L]), and hypogonadism (low testosterone, $2.8 \mathrm{nmol} / \mathrm{L}$ [9.5-28.0 nmol/L]; insufficiently raised follicle-stimulating hormone [FSH], 14.0 IU/L [RI, 1.0-12.0 IU/L]; luteinising hormone [LH] within RI, 3.7 IU/L [RI, 0.6-12 IU/L]). Insulinlike growth factor-1, growth hormone and prolactin levels were within RIs.

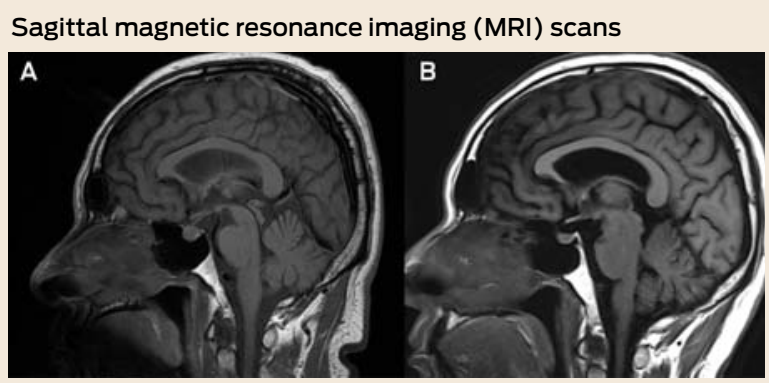

T7-weighted sagittal MRI scans of Patient 1, showing symmetrical pituitary enlargement up to $9.3 \mathrm{~mm}$ in height with superior convexity and loss of the posterior pituitary bright spot at presentation $(\boldsymbol{A})$ consistent with a diagnosis of autoimmune hypophysitis, ${ }^{3}$ followed by normalisation 6 months later $(B)$
Magnetic resonance imaging (MRI) scans of the brain excluded metastatic disease and demonstrated pituitary enlargement without chiasmal or infundibular involvement (Box). The patient was initially commenced on replacement hydrocortisone.

Patient 2 presented with severe headaches and emesis. Morning blood test results demonstrated central hypothyroidism (low TSH, $0.24 \mathrm{mIU} / \mathrm{L}$ [RI, 0.27-4.20 mIU/L]; $\mathrm{FT}_{4^{\prime}}$ $11.4 \mathrm{pmol} / \mathrm{L}$ [RI, $12.0-22.0 \mathrm{pmol} / \mathrm{L}]$; and $\mathrm{FT}_{3}, 2.8 \mathrm{pmol} / \mathrm{L}$ [RI, 3.0$7.8 \mathrm{pmol} / \mathrm{L}])$ and hypogonadism (low testosterone [2.1 nmol/L] and inappropriately normal FSH [8.1 IU/L] and LH [3.9IU/L]). Although morning cortisol and ACTH levels were initially adequate, the patient had been placed on high-dose dexamethasone because of suspicion of brain metastases, which were later excluded by lumbar puncture and brain MRI. The pituitary was not enlarged, but superior convexity was evident.

Both patients received a 3 -week tapering course of prednisolone starting at $30 \mathrm{mg}$ daily and weaned to replacement doses. They received thyroxine and, later, testosterone replacement. Ipilimumab was continued to include the standard fourth and final dose. The endocrine abnormalities persisted in both men at 3-6-month follow-up, despite normalisation of MRI findings (Box). For Patient 2, a subsequent insulintolerance test demonstrated a flat cortisol response consistent with corticotropic insufficiency due to hypophysitis, although suppression from exogenous steroids may have contributed. Both patients experienced tumour progression over the follow-up period.

Ipilimumab was registered by the Therapeutic Goods Administration in 2011 and funded by the Pharmaceutical Benefits Scheme from August 2013. These are the second Australian cases of ipilimumabinduced hypophysitis to be published in the Journal. ${ }^{4}$ Ipilimumab-induced thyroiditis and adrenalitis are common differential diagnoses to consider. ${ }^{5}$ Suprasellar extension is seen frequently, ${ }^{6}$ requiring urgent ophthalmic evaluation. There are no prospective data on whether high-dose steroids improve 
pituitary recovery or abrogate the tumour response to ipilimumab. Our cases demonstrated no shortterm advantage. Interdisciplinary awareness of this condition is required to ensure that replacement glucocorticoids are started promptly on diagnosis and increased during periods of physiological stress, including surgery or chemotherapy.

Sunita MC De Sousa Pituitary Fellow

Georgina V Long Associate Professor of Translational Research and Melanoma Biology ${ }^{2}$

Katherine T Tonks Clinical Research Fellow ${ }^{3}$

1 Department of Endocrinology, St Vincent's Hospital, Sydney, NSW.

2 Melanoma Institute Australia, Sydney, NSW.

3 Diabetes and Metabolism Division, Garvan Institute of Medical Research, Sydney, NSW.

\section{s.desousa@garvan.org.au}

Acknowledgements: We thank Diana Adams of Macarthur Cancer Therapy Centre at Campbelltown Hospital, Sydney, who shared in patient care.

Competing interests: Georgina Long is a consultant adviser to Bristol-Myers Squibb, Merck, Amgen, GlaxoSmithKline, Novartis and Roche.

doi: 10.5694/mjal4.00803

1 Hodi FS, O'Day SJ, McDermott DF, et al. Improved survival with ipilimumab in patients with metastatic melanoma. N Engl J Med 2010; 363: 711-723.

2 Ryder M, Callahan M, Postow MA, et al. Endocrine-related adverse events following ipilimumab in patients with advanced melanoma: a comprehensive retrospective review from a single institution. Endocr Relat Cancer 2014; 21: 371-381.

3 Gutenberg A, Larsen J, Lupi I, et al. A radiologic score to distinguish autoimmune hypophysitis from nonsecreting pituitary adenoma preoperatively. Am J Neuroradiol 2009; 30: 1766-1772.

4 Alexander M, Mellor JD, McArthur G, Kee D. Ipilimumab in pretreated patients with unresectable or metastatic cutaneous, uveal and mucosal melanoma. Med J Aust 2014; 201 49-53.

5 Torino F, Barnabei A, De Vecchis L, et al. Hypophysitis induced by monoclonal antibodies to cytotoxic T lymphocyte antigen 4: challenges from a new cause of a rare disease. Oncologist 2012; 17: 525-535.

6 Blansfield JA, Beck KE, Tran K, et al. Cytotoxic T-lymphocyte-associated antigen-4 blockage can induce autoimmune hypophysitis in patients with metastatic melanoma and renal cancer. J Immunother 2005; 28: 593-598.

\section{Impact of pneumococcal polysaccharide vaccine in people aged 65 years or older}

To THE Editor: Menzies and colleagues promote the 23-valent polysaccharide pneumococcal vaccine (23vPPV) as effective in people aged 65 years or older. ${ }^{1} \mathrm{I}$ believe their data show exactly the opposite. The vaccine appears to be ineffective.

In 2005, a nationally funded vaccination program began with
$23 \mathrm{vPPV}$ for those aged $\geqslant 65$ years and a 7-valent pneumococcal conjugate vaccine $(7 \mathrm{vPCV})$ for children. Subsequently, there was a rapid and dramatic drop in invasive pneumococcal disease in older people, but this was only for disease due to serotypes covered by the childhood $7 \mathrm{vPCV}$. When the effects of the other components of the $23 \mathrm{vPPV}$ are examined, there is no fall but instead a statistically significant increase in invasive infections in older people. ${ }^{1}$

Vaccinating children with highly effective conjugated pneumococcal vaccines is associated with a dramatic decrease in invasive pneumococcal disease not only in children but also in older people. Older people benefit because vaccinated children carry fewer pneumococcal strains and thus pass on fewer invasive strains to parents and grandparents..$^{2-4}$

I think that the only reasonable conclusion from the study by Menzies et al is that the non-conjugated vaccine is ineffective in those aged $\geqslant 65$ years. Its use may perversely have even been associated with an increase in invasive disease.

This clearly shows the superiority and easily measured benefits 
of conjugated pneumococcal vaccines. Why persevere with the unconjugated 23vPPV in older people given its poor or potentially non-existent efficacy? We need to ensure that we have effective pneumococcal vaccines available for older people. This means conjugated pneumococcal vaccines.

Peter J Collignon Professor, Infectious Diseases and Microbiology

Medical School, Australian National University, Canberra, ACT.

Peter.Collignon@act.gov.au

Competing interests: No relevant disclosures.

doi: 10.5694/mjal4.00272

1 Menzies RI, Jayasinghe SH, Krause VL, et al. Impact of pneumococcal polysaccharide vaccine in people aged 65 years or older. Med $J$ Aust 2014; 200: 112-115.

2 Whitney CG, Farley MM, Hadler J, et al. Decline in invasive pneumococcal disease after the introduction of protein-polysaccharide conjugate vaccine. N Engl J Med 2003; 348: 1737-1746.

3 Centers for Disease Control and Prevention. Direct and indirect effects of routine vaccination of children with 7-valent pneumococcal conjugate vaccine on incidence of invasive pneumococcal disease - United States, 1998-2003. MMWR Morb Mortal Wkly Rep 2005; 54: 893-897.

4 Kyaw MH, Lynfield R, Schaffner W, et al. Effect of introduction of the pneumococcal conjugate vaccine on drug-resistant Streptococcus pneumoniae. N Engl J Med 2006; 354: 1455-1463.

IN REPLY: We agree with Collignon that pneumococcal conjugate vaccines (PCVs) are superior to polysaccharide pneumococcal vaccines (PPVs), and that the herd immunity impact of the PCV on older people is impressive. However, we do not agree that our study shows that the PPV is ineffective in older people, or that its use has resulted in an increase in disease.

The increases in disease due to serotypes not contained in the 7 -valent PCV in the $\geqslant 65$-year age group suggest an underlying increase in adults, probably because of serotype replacement driven by the PCV. The fact that these increases were greater in 50-64-year-olds, in whom PPV use was negligible, suggests an attenuating effect from the PPV. The estimate of effectiveness of the PPV in our study was $61 \%$.

Decisions on the PPV's continued use in older people will depend on several factors. These include disease rates in older adults due to serotypes covered by the PPV but not the 13-valent PCV, and any likely additional impact of PCV use in adults over and above the herd immunity impacts from PCV use in children.

Robert I Menzies Deputy Director, Surveillance

Vicki L Krause Director ${ }^{2}$

Peter B McIntyre Director ${ }^{1}$

1 National Centre for Immunisation Research and Surveillance of Vaccine Preventable Diseases, Sydney, NSW.

2 Centre for Disease Control, Department of Health, Darwin, NT.

robert.menzies@health.nsw.gov.au

Competing interests: No relevant disclosures.

doi: 10.5694/mjal4.00399

\section{Managing information overload}

To The Editor: Gee's introduction to a recent issue of the Journal discussed the impact that the overwhelming growth of health information has on doctors. ${ }^{1}$ While it is true that doctors are an important mainstay of advice to patients, health librarians are an important resource to doctors. Health librarians are trained to acquire, organise and

- disseminate credible information resources which enable doctors to find the best evidence to support clinical decision making. ${ }^{2}$

Perhaps the largest exercise to date on the impact of health libraries has been the 2013 Value of library and information services in patient care study in the United States. ${ }^{3}$ The study encompassed 56 libraries serving 118 hospitals with a survey of physicians, residents and nurses involved in either patient care or clinical research. There were 16122 respondents including 5379 physicians, 2123 residents and 6788 nurses. About three-quarters of these respondents reported that they had definitely or probably handled some aspect of patient care differently because of information obtained from libraries. The study found that using health libraries resulted in clinical staff changing the advice given to patients $(48 \%)$, changing diagnosis $(25 \%)$ and changing choice of drugs (33\%), as well as avoiding or reducing the risk of patients misunderstanding their disease $(23 \%)$. $^{3}$

Therefore, as Revere and colleagues highlight, it is important to remember that health librarians can
The fact that these increases were greater in 50-64-yearolds, in whom PPV use was negligible, suggests an attenuating effect from the PPV

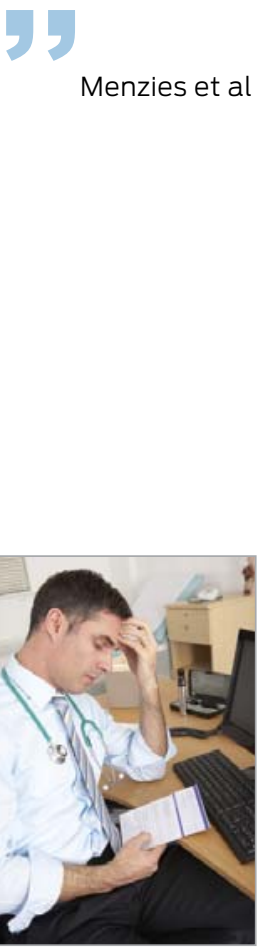


"serve a significant role in helping public health professionals meet their information needs through the development of evidence-based decision support systems, humanmediated expert searching and training in the use [of] information retrieval systems". ${ }^{4}$

Gemma Siemensma Library Manager

Library, Ballarat Health Services, Ballarat, VIC.

\section{gemmas@bhs.org.au}

Competing interests: No relevant disclosures.

doi: 10.5694/mjal4.00678

1 Gee C. Managing information overload [in this issue]. Med J Aust 2014; 200: 365.

2 Holst R, Funk CJ, Adams HS, et al. Vital pathways for hospital librarians: present and future roles. J Med Libr Assoc 2009; 97: 285-292.

3 Marshall JG, Sollenberger J, Easterby-Gannett $S$, et al. The value of library and information services in patient care: results of a multisite study. J Med Libr Assoc 2013; 101: 38-46.

4 Revere D, Turner AM, Madhavan A, et al. Understanding the information needs of public health practitioners: a literature review to inform design of an interactive digital knowledge management system. J Biomed Inform 2007; 40: 410-421

\section{Realising the potential of the post-2015 development agenda for Indigenous health}

To The Editor: The United Nations Millennium Development Goals (MDGs) of 2000 included three health-related goals: reduce child mortality (goal 4), improve maternal health (goal 5) and combat HIV/ AIDS, malaria and other diseases (goal 6). Like many other highincome nations, Australia did not consider its national health priorities subject to the MDG framework. In 2005, the then Aboriginal and Torres Strait Islander Social Justice Commissioner Tom Calma observed that the Australian Government had committed to eradicate poverty through the MDGs"in third world countries by 2015 , but has no similar plans to do so in relation to the extreme marginalisation experienced by Aboriginal and Torres Strait Islander Australians". ${ }^{1}$ In response to the Close the Gap campaign launched in 2007, the government has addressed the health inequalities experienced by Indigenous Australians (with mixed progress reported), and introduced the National Aboriginal and Torres Strait Islander Health Plan 2013-2023. ${ }^{2}$
Global debate around the new post-2015 development goals, which will replace the MDGs when they expire in December 2015, is presently underway, with the intergovernmental Open Working Group on Sustainable Development Goals providing its initial report on the potential goal framework in July 2014 (http://sustainabledevelopment. un.org/focussdgs.html). Although the new goals are not yet finalised, under proposed goal 3 (to ensure healthy lives and promote wellbeing for all at all ages), 13 subgoals are currently being negotiated. The expectation is that the new goals will be universal and applicable to all countries — including highincome nations like Australia — with differentiated targets to redress inequities both between and within countries. Although it is unlikely that there will be any international legal obligation to comply, Australia, as a"good global citizen", may face significant normative pressures to implement domestic policy changes to reflect post-2015 targets. This raises the question of the likely implications for Australian domestic health policy — and for Indigenous health in particular — in light of the new health and development goals. Through post-2015 commitment to overcome domestic and global health and related inequities, the Australian Government will be more accountable on the world stage not only for its Indigenous peoples but also for all people.

\section{Cindy Shannon Pro-Vice-Chancellor (Indigenous} Education)

Claire E Brolan Research Fellow, School of Population Health

Deepa Gajjar Research Officer, School of Population Health

University of Queensland, Brisbane, QLD.

\section{d.gajjar@uq.edu.au}

Acknowledgements: This work forms part of Uptake of evidence to policy: the Indigenous Burden of Disease case study - a collaborative 3-year study between the University of Queensland and the University of New South Wales (National Health and Medical Research Council [NHMRC] grant APP1010534); and Go4Health a research project funded by the European Union Seventh Framework Program (grant HEALTH-Fl-2012-305240) and the Australian Government NHMRC-European Union Collaborative Research Grants (grant 1055138).

Competing interests: No relevant disclosures. doi: 10.5694/mjal4.00059

1 Calma R. Social justice report 2005. (Report No. 3/2005.) Sydney: Human Rights and Equal Opportunity Commission, 2005. http://www. humanrights.gov.au/sites/default/files/ content/social_justice/sj_report/sjreport05/ pdf/SocialJustice2005.pdf (accessed Jul 2014).

2 Australian Government. National Aboriginal and Torres Strait Islander Health Plan 20132023. Canberra: Commonwealth of Australia, 2013. http://www.health.gov.au/internet/ main/publishing.nsf/content/B92E98068048 6C3BCA257BF0001BAF01/\$File/health-plan. pdf (accessed Jul 2014).

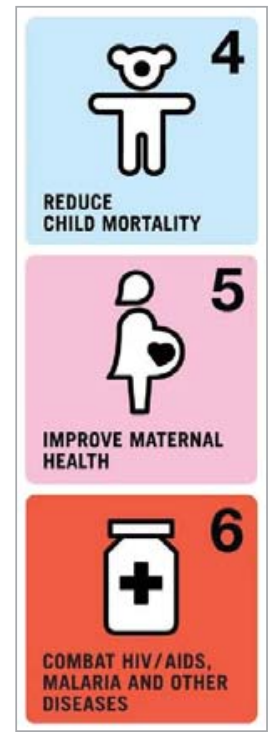

Clarification: In"AIDS, loss and renewal" in the 4 August 2014 issue of the Journal (Med J Aust 2014; 201: 130-132), under the heading"No strangers to suffering", the reference to the murder of Dwayne Jones requires clarification. This section should begin:

So we think of them and of others who have suffered, or are suffering, through irrational, unjust and destructive acts:

- Of Dwayne Jones, murdered in Montego Bay, Jamaica, in July 2013 when she identified as a woman. She was beaten, stabbed, shot and then run over by a car and dumped in a ditch. No one brought to justice.

Australia ... may face significant normative pressures to implement domestic policy changes to reflect post2015 targets

Shannon et al 